\title{
Incidental detection of breast cancer by ${ }^{18 F}$-fluorocholine PET/CT performed for primary hyperparathyroidism
}

\author{
Vlatka Jozanovic ${ }^{1}$ (D), Drazen Huic ${ }^{1,2}$ \\ ${ }^{1}$ Clinical Department of Nuclear Medicine and Radiation Protection, University Hospital Centre Zagreb \\ ${ }^{2}$ The University of Zagreb School of Medicine, Zagreb, Croatia
}

[Received 30 VIII 2021; Accepted 30 XI 2021]

\begin{abstract}
We present a case report of incidental detection of breast cancer in a female patient referred for ${ }^{18} \mathrm{~F}$-fluorocholine (FCH) positron emission tomography/computed tomography (PET/CT) due to primary hyperparathyroidism. This imaging method was recently shown as more sensitive for the detection of metabolically hyperactive parathyroid glands than neck ultrasound and (2-metoksyizobutyloizonitryl labeled with technetium-99m) [99mTc]MIBI. Increased accumulation of FCH was found in the hyperactive parathyroid gland and unexpectedly in the right breast lesion. The surgery confirmed parathyroid adenoma. One month later right upper medial quadrantectomy confirmed breast carcinoma - a combination of invasive ductal carcinoma and intracystic papillary breast carcinoma. To the best of our knowledge, this is the first reporting of simultaneous detection of parathyroid adenoma and breast cancer by using ${ }^{18} \mathrm{~F}$-fluorocholine PET/CT.
\end{abstract}

KEY words: ${ }^{18} \mathrm{~F}$-fluorocholine PET/CT; breast cancer; primary hyperparathyroidism

Nucl Med Rev 2022; 25, 2: xx-67

We report a case of a 75-year old female patient with accidentally diagnosed breast cancer using ${ }^{18} \mathrm{~F}$-fluorocholine PET/CT indicated due to primary hyperparathyroidism. Elevated serum parathyroid hormone (PTH) and calcium levels stood out from the other laboratory parameters. Neck ultrasound examination showed a hypoechoic zone along the upper edge of the right thyroid lobe. Cytological analysis of the punctate verified only thyroid cells without elevated PTH values in the punctate. ${ }^{18} \mathrm{~F}$-fluorocholine (FCH) PET/CT was performed to localize small and hard-to-reach hyperfunctional parathyroid glands. Abnormal cell proliferation and upregulation of choline kinase activity are thought to enhance the accumulation of fluorocholine and increase the efficiency of detecting such abnormalities. Thanks to these features of this radiopharmaceutical, the detection of enhanced cell proliferation ,

Correspondence to: Vlatka Jozanovic, Clinical Department of Nuclear Medicine and Radiation Protection, University Hospital Centre Zagreb Kispaticeva 12, 10000 Zagreb, Croatia,

tel.: 0038 5981807705, e-mail: vlatka.jozanovic@gmail.com which is typical for neoplasms, has become more frequent. Imaging of neck and thoracic regions was performed 20 minutes after intravenous administration of $119 \mathrm{MBq}(3.2 \mathrm{mCi})$ of $\left[{ }^{18} \mathrm{~F}\right] \mathrm{FCH}$. Increased metabolic activity was found in the parathyroid gland with axial dimensions of $0.9 \times 1.0 \mathrm{~cm}$ localized behind the upper third of the right thyroid lobe (Fig. 1). In addition, a metabolically active isodense lesion with irregular edges measuring $1.4 \times 1.0$ $\mathrm{cm}$ located in the upper medial quadrant of the right breast highly suspicious of the neoplasm was verified (Fig. 2). Resection of the described hyperfunctional parathyroid gland was performed and the pathohistological diagnosis corresponded to the adenoma. Postoperative calcium values returned to normal, while PTH levels were slightly elevated. Shortly afterwards, the patient underwent upper medial quadrantectomy of the right breast. The final pathohistological diagnosis confirmed the findings of cytological puncture and percutaneous biopsy that it was combination of invasive ductal carcinoma and intracystic papillary breast carcinoma. Since the diagnostic potential of ${ }^{18} \mathrm{~F}$-fluorocholine has already been investigated in cancers of stomach, prostate, breast as well as melanoma or lymphoma, the possibility of concomitant neoplasms should always be considered when imaging with $\left[{ }^{18} \mathrm{~F}\right] \mathrm{FCH}$. 


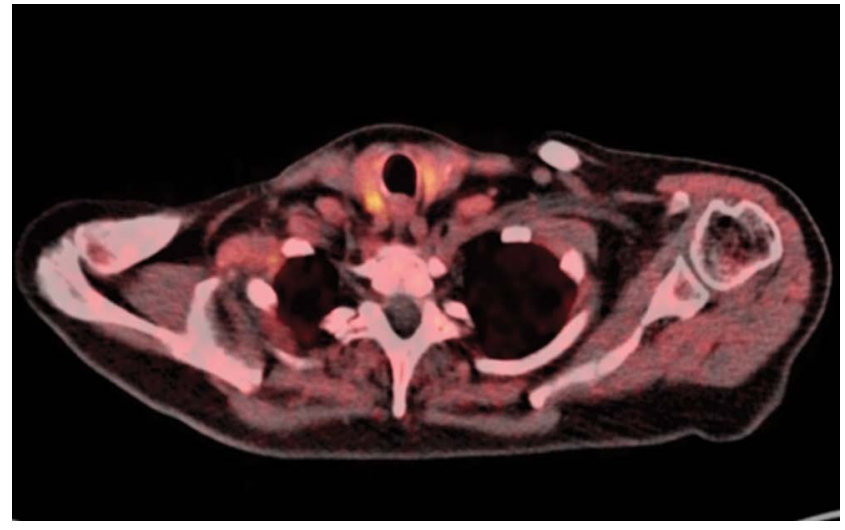

Figure 1. $\left[{ }^{18} \mathrm{~F}\right] \mathrm{FCH} \mathrm{PET} / \mathrm{CT}$ showed increased uptake in the parathyroid gland localized behind the right thyroid lobe

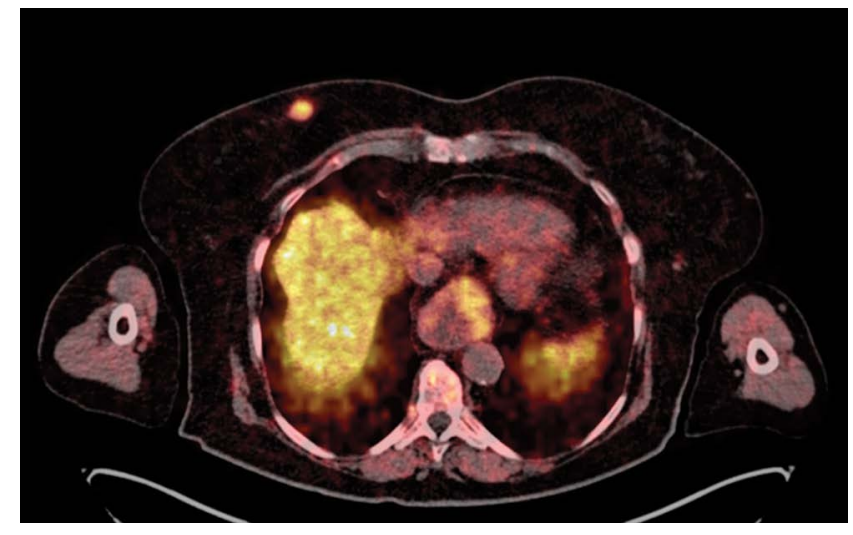

Figure 2. A metabolically active lesion in the right breast highly suspicious of the neoplasm 